\title{
QUASI-EXACTLY SOLVABLE POTENTIALS WITH THREE KNOWN EIGENSTATES
}

\author{
T. V. Kuliy* and V. M. Tkachuk** \\ Ivan Franko Lviv State University, Chair of Theoretical Physics \\ 12 Drahomanov Str., Lviv UA-290005, Ukraine \\ *E-mail: Kuliy@KTF.Franko.Lviv.UA, ${ }^{* *}$ E-mail: Tkachuk@KTF.Franko.Lviv.UA
}

August 20, 2018

\begin{abstract}
We propose a new SUSY method for the generation of the quasi-exactly solvable (QES) potentials with three known eigenstates. New QES potentials and corresponding energy levels and wave functions of the ground state and two lowest excited state are obtained. A possibility to construct families of exactly solvable non-singular potentials which are SUSY partners of the well known ones is shown.

Key words: supersymmetry, quantum mechanics, quasi-exactly solvable potentials.
\end{abstract}

PACS number(s): 03.65.-w; 11.30.Pb

\section{Introduction}

About twenty years ago an interesting class of the so-called quasi-exactly solvable (QES) potentials for which a finite number of eigenstates is analytically known was introduced [1 [4. Nowadays the QES problems attract much attention [5 19]. Several methods for generation of QES potentials have been worked out and as a result many QES potentials have been established. For example three different methods based respectively on a polynomial anzatz for wave functions, point canonical transformation and supersymmetric (SUSY) quantum mechanics are described in the paper [12].

The SUSY is very useful tool for study of exactly solvable potentials. Note the papers [20,21] where SUSY procedure for constructing Hamiltonians either with identical spectra or with identical spectra, apart from a missing ground state, was given. This procedure may be repeated again and again to generate hierarchies of Hamiltonians whose spectra are related to each other. Some recent papers on this subject one can find in [22] 25]. For review of SUSY quantum mechanics see [26, 27]

At the first time the SUSY method for constructing QES potentials was used in [10 12]. The idea of this method is the following. Starting from some initial QES potential with $n+1$ known eigenstates and using the properties of the unbroken SUSY one obtains the supersymmetric partner potential which is a new QES one with $n$ known eigenstates.

In our previous paper [28] we proposed a new SUSY method for generating QES potentials with explicitly known two eigenstates. This method in contrast to the the 
papers [10 12] does not require the knowledge of the initial QES potential for generating of a new QES one. In the present paper we develop this SUSY method for constructing QES potentials with explicitly known three eigenstates.

\section{Witten's model of SUSY quantum mechanics}

Let us first take a look at the Witten's model of SUSY quantum mechanics. The algebra of SUSY in this case satisfies the following permutation relations

$$
\begin{aligned}
& \left\{Q^{+}, Q^{-}\right\}=H, \\
& {\left[Q^{ \pm}, H\right]=0,} \\
& \left(Q^{ \pm}\right)^{2}=0,
\end{aligned}
$$

where the supercharges read

$$
Q^{+}=B^{-} \sigma^{+}, Q^{-}=B^{+} \sigma^{-}
$$

$\sigma^{ \pm}$are the Pauli matrices,

$$
B^{ \pm}=\frac{1}{\sqrt{2}}\left(\mp \frac{d}{d x}+W(x)\right)
$$

$W(x)$ is the superpotential. The Hamiltonian consists of a pair of standard Schrödinger operators $H_{ \pm}$

$$
H=\left(\begin{array}{cc}
H_{+} & 0 \\
0 & H_{-}
\end{array}\right)
$$

where

$$
H_{ \pm}=B^{\mp} B^{ \pm}=-\frac{1}{2} \frac{d^{2}}{d x^{2}}+V_{ \pm}(x),
$$

$V_{ \pm}(x)$ are the so-called SUSY partner potentials

$$
V_{ \pm}(x)=\frac{1}{2}\left(W^{2}(x) \pm W^{\prime}(x)\right), \quad W^{\prime}(x)=\frac{d W(x)}{d x}
$$

Consider the equation for the energy spectrum

$$
H_{ \pm} \psi_{n}^{ \pm}(x)=E_{n}^{ \pm} \psi_{n}^{ \pm}(x), \quad n=0,1,2, \ldots
$$

The Hamiltonians $H_{+}$and $H_{-}$have the same energy spectrum except the zero energy ground state which exists in the case of the unbroken SUSY. This leads to two-fold degeneracy of the energy spectrum of $H$ except for the unique zero energy ground state. 
Only one of the Hamiltonians $H_{ \pm}$has the zero energy eigenvalue. We shall use the convention that the zero energy eigenstate belongs to $H_{-}$. Due to the factorization of the Hamiltonians $H_{ \pm}$(see (5)) the ground state for $H_{-}$satisfies the equation

$$
B^{-} \psi_{0}^{-}(x)=0
$$

with the solution

$$
\psi_{0}^{-}(x)=C \exp \left(-\int W(x) d x\right),
$$

here $C$ is the normalization constant. From the normalization condition it follows that

$$
\operatorname{sign}(W(x))= \pm 1
$$

when $x \rightarrow \pm \infty$.

The eigenvalues and eigenfunctions of the Hamiltonians $H_{+}$and $H_{-}$are related by the SUSY transformations

$$
\begin{aligned}
& E_{n+1}^{-}=E_{n}^{+}, \quad E_{0}^{-}=0, \\
& \psi_{n+1}^{-}(x)=\frac{1}{\sqrt{E_{n}^{+}}} B^{+} \psi_{n}^{+}(x), \\
& \psi_{n}^{+}(x)=\frac{1}{\sqrt{E_{n+1}^{-}}} B^{-} \psi_{n+1}^{-}(x) .
\end{aligned}
$$

The two properties of the unbroken SUSY quantum mechanics, namely, a two-fold degeneracy of the spectrum and the existence of the zero energy ground state are used for the exact calculation of the energy spectrum and wave functions (see reviews [26, 27]).

\section{SUSY constructing QES potentials}

We shall study the Hamiltonian $H_{-}$the ground state of which is given by (9). Let us consider the SUSY partner of $H_{-}$, i.e. the Hamiltonian $H_{+}$. If we calculate the ground state of $H_{+}$we immediately find the first excited state of $H_{-}$using the degeneracy of the spectrum of the SUSY Hamiltonian and transformations (11), (12), (13). In order to calculate the ground state of $H_{+}$let us rewrite it in the following form

$$
H_{+}=H_{-}^{(1)}+\epsilon, \quad \epsilon>0
$$

where

$$
\begin{aligned}
& H_{-}^{(1)}=B_{1}^{+} B_{1}^{-}, \\
& B_{1}^{ \pm}=\frac{1}{\sqrt{2}}\left(\mp \frac{d}{d x}+W_{1}(x)\right),
\end{aligned}
$$


$\epsilon$ is the energy of the ground state of $H_{+}$since $H_{-}^{(1)}$ has zero energy ground state.

As we see from (14), (15) the ground state wave function of $H_{+}$is also the ground state wave function of $H_{-}^{(1)}$ and it satisfies the equation

$$
B_{1}^{-} \psi_{0}^{+}(x)=0
$$

The solution of this equation is

$$
\psi_{0}^{+}(x)=C \exp \left(-\int W_{1}(x) d x\right)
$$

Then using SUSY transformation (12) we can easily calculate the wave function of the first excited state of $H_{-}$. Repeating the described procedure for $H_{-}^{(1)}$ we obtain the second excited state for $H_{-}$. Continuing this procedure $N$ times we obtain $N$ excited states. This procedure is well known in SUSY quantum mechanics [20, 21] (see also reviews [26, 27]). The wave functions and corresponding energy levels read

$$
\begin{aligned}
\psi_{n}^{-}(x) & =C_{n}^{-} B_{0}^{+} \ldots B_{n-2}^{+} B_{n-1}^{+} \exp \left(-\int W_{n}(x) d x\right), \\
E_{n}^{-} & =\sum_{i=0}^{n-1} \epsilon_{i},
\end{aligned}
$$

where $n=1,2, \ldots, N$. In our notations $\epsilon_{0}=\epsilon, B_{0}^{ \pm}=B^{ \pm}, W_{0}(x)=W(x)$. Operators $B_{n}^{ \pm}$ are given by (3) with the superpotentials $W_{n}(x)$. The equation (14) rewritten for $N$ steps

$$
H_{+}^{(n)}=H_{-}^{(n+1)}+\epsilon_{n}, \quad n=0,1, \ldots, N-1
$$

leads to the set of equations for superpotentials

$$
W_{n}^{2}(x)+W_{n}^{\prime}(x)=W_{n+1}^{2}(x)-W_{n+1}^{\prime}(x)+2 \epsilon_{n}, \quad n=0,1, \ldots, N-1 .
$$

Previously this set of equations for $W_{n}(x)$ was solved in the special cases of the so-called shape-invariant potentials [29] and self-similar potentials for arbitrary $N$ (see review [30]). For $N=1$ one can easily obtain a general solution of (21) without restricting ourselves to shape-invariant or self-similar potentials. This solution was obtained in [31] in the context of parasupersymmetric quantum mechanics.

In our recent paper [28] we construct nonsingular solution of (21) for $N=1$ in order to obtain nonsingular QES potentials with two known eigenstates.

In present paper we use the method proposed in [28] to solve the set of equations (21) for $N=2$. It give us the possibility to obtain the general expression for wave functions and energy levels of QES potentials with three explicitly known eigenstates.

Let us introduce new functions

$$
W_{+}^{(n)}(x)=W_{n+1}(x)+W_{n}(x)
$$




$$
W_{-}^{(n)}(x)=W_{n+1}(x)-W_{n}(x), \quad n=0,1, \ldots, N-1 .
$$

Then equations (21) read

$$
W_{+}^{\prime(n)}(x)=W_{-}^{(n)}(x) W_{+}^{(n)}(x)+2 \epsilon_{n} .
$$

One can easily solve these equations with respect to the functions $W_{-}^{(n)}(x)$ obtaining the following expressions for superpotentials

$$
\begin{aligned}
& W_{n}(x)=\frac{1}{2}\left(W_{+}^{(n)}(x)-\frac{W_{+}^{\prime(n)}(x)-2 \epsilon_{n}}{W_{+}^{(n)}(x)}\right), \\
& W_{n+1}(x)=\frac{1}{2}\left(W_{+}^{(n)}(x)+\frac{W_{+}^{\prime(n)}(x)-2 \epsilon_{n}}{W_{+}^{(n)}(x)}\right),
\end{aligned}
$$

which lead obviously to the following set of equations for functions $W_{+}^{(n)}(x)$

$$
\begin{aligned}
& W_{+}^{(n)}(x)+\frac{W_{+}^{\prime(n)}(x)-2 \epsilon_{n}}{W_{+}^{(n)}(x)}=W_{+}^{(n+1)}(x)+\frac{W_{+}^{\prime(n+1)}(x)-2 \epsilon_{n+1}}{W_{+}^{(n+1)}(x)}, \\
& n=0,1, \ldots, N-2 .
\end{aligned}
$$

Thus the set of $N$ equations (21) is reduced to the set of $N-1$ equations (25). In the simplest case $N=1$ equation (25) is absent and relations (24) express just a general solution of equation (21). To obtain a general solution of set (21) in the case $N=2$ we have to solve one equation:

$$
W_{+}(x)+\frac{W_{+}^{\prime}(x)-2 \epsilon}{W_{+}(x)}=\tilde{W}_{+}(x)-\frac{\tilde{W}_{+}^{\prime}(x)-2 \epsilon_{1}}{\tilde{W}_{+}(x)}
$$

where we have introduced for short the notation

$$
W_{+}(x) \equiv W_{+}^{(0)}(x), \quad \tilde{W}_{+}(x) \equiv W_{+}^{(1)}(x)
$$

It is easily to rewrite this equation as follows

$$
W_{+}(x) \tilde{W}_{+}(x)\left(\tilde{W}_{+}(x)-W_{+}(x)\right)-\left(W_{+}(x) \tilde{W}_{+}(x)\right)^{\prime}+2\left(\epsilon_{1} W_{+}(x)+\epsilon \tilde{W}_{+}(x)\right)=0,
$$

or

$$
U(x)\left(\frac{U(x)}{W_{+}(x)}-W_{+}(x)\right)-U^{\prime}(x)+2\left(\epsilon_{1} W_{+}(x)+\epsilon \frac{U(x)}{W_{+}(x)}\right)=0,
$$


where we have introduced the function

$$
U(x)=W_{+}(x) \tilde{W}_{+}(x) .
$$

We again came to the Riccati equation with respect to $U(x)$. On the other hand it is an algebraic equation with respect to $W_{+}(x)$. Thus we can start from arbitrary function $U(x)$ to construct the functions $W_{+}(x)$ and $\tilde{W}_{+}(x)$ which take the form

$$
\begin{gathered}
W_{+}(x)=\frac{2 U(x)(U(x)+2 \epsilon)}{U^{\prime}(x)(1+\mathcal{R}(x))}, \\
\tilde{W}_{+}(x)=\frac{U^{\prime}(x)(1+\mathcal{R}(x))}{2(U(x)+2 \epsilon)},
\end{gathered}
$$

where

$$
\begin{aligned}
& \mathcal{R}(x)= \pm R(x), \\
& R(x)=\sqrt{1+4 \frac{U(x)(U(x)+2 \epsilon)\left(U(x)-2 \epsilon_{1}\right)}{U^{\prime}(x)^{2}} .}
\end{aligned}
$$

We mean square root $R(x)$ as positively defined value, while function $\mathcal{R}(x)$ can be chosen in the form of $R(x)$ or $-R(x)$ within different intervals separated by zeros of function $R(x)$. Note that just the possibility to choose different signs allows us, as will be shown in the section 5, to construct in a simple way new exactly solvable potentials using the known ones.

Now we can obtain three consequent superpotentials $W(x), W_{1}(x)$ and $W_{2}(x)$ using the relations (24). Then using (18) and (19) we obtain the energy levels and the wave functions of the first and the second excited states for $H_{-}$

$$
\begin{aligned}
& E_{1}^{-}=\epsilon, \quad E_{2}^{-}=\epsilon+\epsilon_{1}, \\
& \psi_{1}^{-}(x)=C_{1} W_{+}(x) \exp \left(-\int W_{1}(x) d x\right), \\
& \psi_{2}^{-}(x)=C_{2}\left(\left(W(x)+W_{2}(x)\right) \tilde{W}_{+}(x)-\tilde{W}_{+}^{\prime}\right) \exp \left(-\int W_{2}(x) d x\right) .
\end{aligned}
$$

The superpotentials $W_{1}(x)$ and $W_{2}(x)$ must satisfy the same condition (10) as $W(x)$. It leads to the same limitations for the functions $W_{+}(x)$ and $\tilde{W}_{+}(x)$. Both must be positive at infinity, negative at minus infinity and therefore each of them must posses at least one zero. Let us consider at first continuous superpotentials. As is seen from the (24) to avoid singularity of superpotentials the functions $W_{+}(x)$ and $\tilde{W}_{+}(x)$ ought have each only one zero 28

$$
W_{+}\left(x_{0}\right)=0, \quad \tilde{W}_{+}\left(\tilde{x}_{0}\right)=0
$$


at which they must satisfy the condition

$$
W_{+}^{\prime}\left(x_{0}\right)=2 \epsilon, \quad \tilde{W}_{+}^{\prime}\left(\tilde{x}_{0}\right)=2 \epsilon_{1} .
$$

Thus we have a number of limitations in the choice of function $U(x)$ as a product of $W_{+}(x)$ and $\tilde{W}_{+}(x)$. There are two different possibilities of the choice of the function $U(x)$. Either $x_{0}=\tilde{x}_{0}$ that provides $U(x)$ to have only one second-order zero point and to be positive at all the rest number line

$$
\begin{cases}U\left(x_{0}\right)=0, & U^{\prime}\left(x_{0}\right)=0, \quad U^{\prime \prime}\left(x_{0}\right)>0 \\ U(x)>0, & x \neq x_{0}\end{cases}
$$

or $x_{0} \neq \tilde{x}_{0}$ and therefore $U(x)$ has two zero points and changes its sign as follows

$$
\begin{cases}U(x)<0, & x \in\left(\min \left[x_{0}, \tilde{x}_{0}\right], \max \left[x_{0}, \tilde{x}_{0}\right]\right), \\ U\left(x_{0}\right)=U\left(\tilde{x}_{0}\right)=0, & U^{\prime}\left(\min \left[x_{0}, \tilde{x}_{0}\right]\right)<0, \quad U^{\prime}\left(\max \left[x_{0}, \tilde{x}_{0}\right]\right)>0, \\ U(x)>0, & x \notin\left[\min \left[x_{0}, \tilde{x}_{0}\right], \max \left[x_{0}, \tilde{x}_{0}\right]\right] .\end{cases}
$$

The sign of the function $\mathcal{R}(x)$ in the expressions (31) for the functions $W_{+}(x)$ and $\tilde{W}_{+}(x)$ should be chosen in a such way to ensure smoothness of these functions and an existence of one zero for each of them. A full analysis of the conditions which must satisfy the function $U(x)$ to provide continuous superpotentials is rather boring and includes consideration of the behaviour of the functions $W_{+}(x)$ and $\tilde{W}_{+}(x)$ nearby possible zeroes of the expressions

$$
U(x)+2 \epsilon, \quad U(x)-2 \epsilon_{1}, \quad U^{\prime}(x), \quad R(x),
$$

which is crucial for the continuity of the final superpotentials $W(x), W_{1}(x)$ and $W_{2}(x)$.

We shall consider more closely the simplest case of functions $U(x)$ which has one zero point and satisfies the conditions (37). The other condition which the function $U(x)$ must satisfy is a consequence of the conditions (36) that connect the derivatives of the functions $W_{+}(x)$ and $\tilde{W}_{+}(x)$ with the energies $\epsilon$ and $\epsilon_{1}$. One can easily find that

$$
\begin{aligned}
U^{\prime \prime}\left(x_{0}\right) & =W_{+}^{\prime \prime}\left(x_{0}\right) \tilde{W}_{+}\left(x_{0}\right)+2 W_{+}^{\prime}\left(x_{0}\right) \tilde{W}_{+}^{\prime}\left(x_{0}\right)+W_{+}\left(x_{0}\right) \tilde{W}_{+}^{\prime \prime}\left(x_{0}\right) \\
& =2 W_{+}^{\prime}\left(x_{0}\right) \tilde{W}_{+}^{\prime}\left(x_{0}\right)=8 \epsilon \epsilon_{1} .
\end{aligned}
$$

The other obvious condition imposed on the function $U(x)$ is positivity of the expression under the square root of the function $R(x)$ (33)

$$
\frac{U^{\prime}(x)^{2}+4 U(x)(U(x)+2 \epsilon)\left(U(x)-2 \epsilon_{1}\right)}{U^{\prime}(x)^{2}} \geq 0 .
$$

One can easily check that $R\left(x_{0}\right)=0$. Let us consider the case when point $x_{0}$ is unique zero of the function $R(x)$. Then the only way to construct non-singular potentials is to chose function $\mathcal{R}(x)=R(x)$ over all the line in (32). Besides we shall require the function $R(x)$ to be smooth in the vicinity of point $x_{0}$ to avoid cusps of functions $W_{+}(x)$ and 
$\tilde{W}_{+}(x)$ at this point. Note that such cusps would result in $\delta$-like singularities of the final potential $V_{-}(x)$.

Thus we obtain one of possible sets of conditions for the function $U(x)$ allowing to construct non-singular QES potentials with three known eigenstates

$$
\begin{aligned}
& U(x)>0 \quad \forall x \neq x_{0}, \\
& U\left(x_{0}\right)=0, \quad U^{\prime}\left(x_{0}\right)=0, \quad U^{\prime \prime}\left(x_{0}\right)=8 \epsilon \epsilon_{1}, \quad U^{\prime \prime \prime}\left(x_{0}\right)=0, \\
& U^{(4)}\left(x_{0}\right)=64 \epsilon \epsilon_{1}\left(\epsilon_{1}-\epsilon\right), \quad U^{(5)}\left(x_{0}\right)=0, \quad \frac{U^{(6)}\left(x_{0}\right)}{8 \epsilon \epsilon_{1}} \geq 32\left(2 \epsilon_{1}^{2}-13 \epsilon \epsilon_{1}+2 \epsilon^{2}\right), \\
& R(x)>0 \quad \forall x \neq x_{0} .
\end{aligned}
$$

Note that the most of exactly solvable potentials which are continuous over all the line satisfy these conditions.

\section{Examples}

One can easily check that the simplest functions $U(x)$ yield us the well-known potentials. For example starting from $U(x)=4 \epsilon \epsilon_{1} x^{2}$ at $\epsilon_{1}=\epsilon$ we get harmonic oscillator potential. Another simple function $U(x)=4 \epsilon \epsilon_{1} \tanh ^{2} x$ at $\epsilon_{1}=\epsilon-1$ leads us to the well-known exactly solvable Rosen-Morse potential. One more simple example $U(x)=4 \epsilon \epsilon_{1} \sinh ^{2} x$ at $\epsilon_{1}=\epsilon+\frac{1}{2}$ reproduces the special case of the well-known quasi exactly solvable Razavy potential [3].

Let us consider more complicated examples leading to new QES potentials. We shall start from the function

$$
U(x)=4 \epsilon \epsilon_{1} x^{2} \frac{1+a^{2} x^{2}}{1+b^{2} x^{2}},
$$

where $a$ and $b$ are real parameters.

Due to the conditions for $U^{(4)}\left(x_{0}\right)$ and $U^{(6)}\left(x_{0}\right)$ from (42) we get

$$
a^{2}=b^{2}+\frac{2}{3}\left(\epsilon_{1}-\epsilon\right)
$$

and

$$
\frac{13}{4} \frac{\epsilon}{b^{2}}-\frac{15}{8}-\frac{3}{8} \sqrt{\Delta} \leq \frac{\epsilon_{1}}{b^{2}} \leq \frac{13}{4} \frac{\epsilon}{b^{2}}-\frac{15}{8}+\frac{3}{8} \sqrt{\Delta}
$$

respectively; here

$$
\Delta=25-60 \frac{\epsilon}{b^{2}}+68\left(\frac{\epsilon}{b^{2}}\right)^{2} .
$$


Because $a$ and $b$ are real numbers it follows from (44) that

$$
\frac{\epsilon}{b^{2}}-\frac{3}{2} \leq \frac{\epsilon_{1}}{b^{2}}
$$

It is easily to check that

$$
\frac{13}{4} \frac{\epsilon}{b^{2}}-\frac{15}{8}-\frac{3}{8} \sqrt{\Delta} \leq \frac{\epsilon}{b^{2}}-\frac{3}{2}
$$

and therefore (45) together with (44) lead to the following inequality

$$
\frac{\epsilon}{b^{2}}-\frac{3}{2} \leq \frac{\epsilon_{1}}{b^{2}} \leq \frac{13}{4} \frac{\epsilon}{b^{2}}-\frac{15}{8}+\frac{3}{8} \sqrt{\Delta}
$$

Thus in order to obtain nonsingular potentials the parameters of function (43) must satisfy conditions (44) and (49). Besides we obviously must require the parameters $\epsilon$ and $\epsilon_{1}$ to be positive.

We shall omit the general expression for the potential $V_{-}(x)$ as it is huge and rather useless. It is easily to show that there are only two sets of positive $\epsilon$ and $\epsilon_{1}$ allowing to resolve the root in the function $R(x)$ (Cases 1 and 2) and therefore to simplify significantly the final expressions. The other simplified expression (Case 3) we shall obtain putting $a=0$ that corresponds to the lowest value of the parameter $\epsilon_{1}$ in inequality (49).

\subsection{Case 1}

In the case

$$
\epsilon=\frac{3}{2} b^{2}, \quad \epsilon_{1}=\left(\frac{3}{2}+\sqrt{3}\right) b^{2}
$$

square root in $R(x)$ can be resolved and we obtain the potential

$$
\begin{aligned}
V_{-}(x)= & \left(-\frac{9}{4}-2 \sqrt{3}+3\left(\frac{7}{8}+\frac{\sqrt{3}}{2}\right) b^{2} x^{2}+\right. \\
& \left.\frac{3-\sqrt{3}}{2-\sqrt{3}+b^{2} x^{2}}+\frac{7 \sqrt{3}-12}{\left(2-\sqrt{3}+b^{2} x^{2}\right)^{2}}\right) b^{2}
\end{aligned}
$$

and the eigenfunctions

$$
\begin{aligned}
& \psi_{0}(x)=C e^{-\frac{(3+2 \sqrt{3})}{4} b^{2} x^{2}}\left(2-\sqrt{3}+b^{2} x^{2}\right)^{\frac{(3-\sqrt{3})}{2}}, \\
& \psi_{1}(x)=C_{1} e^{-\frac{(3+2 \sqrt{3})}{4} b^{2} x^{2}}\left(2-\sqrt{3}+b^{2} x^{2}\right)^{\frac{(\sqrt{3}-1)}{2}} x, \\
& \psi_{2}(x)=C_{2} e^{-\frac{(3+2 \sqrt{3})}{4} b^{2} x^{2}}\left(2-\sqrt{3}+b^{2} x^{2}\right)^{\frac{(\sqrt{3}-1)}{2}}\left(2-\sqrt{3}-b^{2} x^{2}\right)
\end{aligned}
$$


corresponding to the three lowest levels

$$
E_{0}^{-}=0, \quad E_{1}^{-}=\frac{3}{2} b^{2}, \quad E_{2}^{-}=(3+\sqrt{3}) b^{2} .
$$

To simplify these expressions let us make the substitution

$$
b^{2}=(2-\sqrt{3}) c^{2}
$$

Thus we get

$$
\begin{aligned}
& V_{-}(x)=c^{2}\left(\frac{3}{8} c^{2} x^{2}+\frac{3-\sqrt{3}}{1+c^{2} x^{2}}+\frac{2 \sqrt{3}-3}{\left(1+c^{2} x^{2}\right)^{2}}-\frac{7 \sqrt{3}}{4}+\frac{3}{2}\right), \\
& E_{0}^{-}=0, E_{1}^{-}=3\left(1-\frac{\sqrt{3}}{2}\right) c^{2}, \quad E_{2}^{-}=(3-\sqrt{3}) c^{2}, \\
& \psi_{0}^{-}(x)=C e^{-\frac{\sqrt{3}}{4} c^{2} x^{2}}\left(1+c^{2} x^{2}\right)^{\frac{(3-\sqrt{3})}{2}}, \\
& \psi_{1}^{-}(x)=C_{1} e^{-\frac{\sqrt{3}}{4} c^{2} x^{2}}\left(1+c^{2} x^{2}\right)^{\frac{(\sqrt{3}-1)}{2}} x \\
& \psi_{2}^{-}(x)=C_{2} e^{-\frac{\sqrt{3}}{4} c^{2} x^{2}}\left(1+c^{2} x^{2}\right)^{\frac{(\sqrt{3}-1)}{2}}\left(1-c^{2} x^{2}\right) .
\end{aligned}
$$

It is worth to note that the obtained potential (57) is double-well one. As well known, double-well potentials has been used extensively to model a wide range of natural phenomena.

\subsection{Case 2}

The other set of positive $\epsilon$ and $\epsilon_{1}$ resolving the root in the function $R(x)$ reads

$$
\epsilon=\frac{3}{2} b^{2}, \quad \epsilon_{1}=\frac{1}{2} b^{2}
$$

It leads to the well-known supersymmetric partner of a harmonic oscillator 22, 23, 33

$$
V_{-}(x)=\frac{3 b^{2}}{4}+\frac{b^{4} x^{2}}{8}-\frac{4 b^{2}}{\left(1+b^{2} x^{2}\right)^{2}}+\frac{2 b^{2}}{1+b^{2} x^{2}}
$$

This potential is exactly solvable although we have got it using the procedure for constructing QES potentials with three exactly known eigenstates. We will also obtain the same potential in the section 5 using the other procedure allowing to construct new exactly solvable potentials (see (81)). 


\subsection{Case 3}

Let us consider another particular case of the potential under consideration which is rather simple. We put $a=0$ to reduce the function $U(x)$ (43) to the form

$$
U(x)=4 \epsilon \epsilon_{1} \frac{x^{2}}{1+b^{2} x^{2}}
$$

Due to the relation (44) we obtain the connection between the energies $\epsilon$ and $\epsilon_{1}$

$$
\epsilon=\epsilon_{1}+\frac{3}{2} b^{2}
$$

Note that in accordance with relation (49) such a choice of parameters corresponds to the minimal value of $\epsilon_{1}$ at given $\epsilon$ and $b$. It leads directly to the potential

$$
\begin{aligned}
\frac{V_{-}(x)}{b^{2}}= & \frac{\left(\alpha^{2}+3 \alpha+1\right)^{2}}{8 \alpha(\alpha+1)}+\frac{1}{1+b^{2} x^{2}}-\frac{2}{\left(1+b^{2} x^{2}\right)^{2}}-\frac{2}{\rho(x)\left(1+b^{2} x^{2}\right)^{2}} \\
& -\frac{\alpha^{2}+\alpha-1}{\rho(x)\left(1+b^{2} x^{2}\right)}-\frac{\left(\alpha^{2}+\alpha+1\right)^{3}}{8 \alpha(\alpha+1) \rho^{2}(x)}+\frac{\left(\alpha^{2}+\alpha+1\right)^{2}}{4 \rho^{3}(x)}
\end{aligned}
$$

and the following eigenfunctions

$$
\begin{aligned}
& \psi_{0}^{-}(x)=C_{0} \frac{\rho(x)+\alpha}{\rho(x)-1} e^{-\frac{1}{2} \rho(x)\left(1+\frac{1}{\alpha}+\frac{1}{\alpha+1}\right)}, \\
& \psi_{1}^{-}(x)=C_{1} \frac{x}{\rho(x)-1} e^{-\frac{1}{2} \rho(x)\left(1-\frac{1}{\alpha}+\frac{1}{\alpha+1}\right)}, \\
& \psi_{2}^{-}(x)=C_{2} \frac{\rho(x)-\alpha-1}{\rho(x)-1} e^{-\frac{1}{2} \rho(x)\left(1-\frac{1}{\alpha}-\frac{1}{\alpha+1}\right)},
\end{aligned}
$$

corresponding to the levels

$$
E_{0}^{-}=0, \quad E_{1}^{-}=\epsilon_{1}+\frac{3}{2} b^{2}, \quad E_{2}^{-}=2 \epsilon_{1}+\frac{3}{2} b^{2},
$$

where

$$
\rho(x)=\sqrt{1+\alpha(\alpha+1)\left(1+b^{2} x^{2}\right)}, \quad \alpha=1+2 \frac{\epsilon_{1}}{b^{2}} .
$$

Obtained potential (66) has one minimum at $x=0$ and tends to constant for $x \rightarrow \pm \infty$. For the case $\alpha>1$ all the obtained wave functions (67 69) are square integrable and there exist at least three bound state in the well. For $(\sqrt{5}-1) / 2<\alpha \leq 1$ only two lower wave functions $(67),(68)$ are square integrable and we have just two bound states, while for $\alpha \leq(\sqrt{5}-1) / 2$ only the ground state wave function (67) remains square integrable and 
the potential has only one bound state.

\section{Constructing exactly solvable potentials}

Although we have developed our scheme to construct new QES potentials it seems to be also of use for constructing SUSY partner potentials for the exactly solvable ones. Let us start from some exactly solvable potential $V_{-}(x)$

$$
V_{-}(x)=\frac{1}{2}\left(W^{2}(x)-W^{\prime}(x)\right)
$$

for which we know three first superpotentials $W(x), W_{1}(x)$ and $W_{2}(x)$ satisfying set of equations (21). One can easily construct the functions $W_{+}(x)=W(x)+W_{1}(x)$, $\tilde{W}_{+}(x)=W_{1}(x)+W_{2}(x)$ and $U(x)=W_{+}(x) \tilde{W}_{+}(x)$. Substituting function $U(x)$ into the expressions (31) we can obviously reproduce the functions $W_{+}(x)$ and $\tilde{W}_{+}(x)$ choosing corresponding signs in the function (32) which we shall denote as $\mathcal{R}_{0}(x)$. Beside the functions $W_{+}(x)$ and $\tilde{W}_{+}(x)$ we can obviously obtain another pair of functions $\mathcal{W}_{+}(x)$ and $\tilde{\mathcal{W}}_{+}(x)$ given by the same expressions (31) with the only difference that here we choose the function (32) with the opposite sign to $\mathcal{R}_{0}(x)$,

$$
\mathcal{R}(x)=-\mathcal{R}_{0}(x)
$$

The new functions $\mathcal{W}_{+}(x)$ and $\tilde{\mathcal{W}}_{+}(x)$ satisfy the same equation (26) and just they allow us to construct new exactly solvable potentials.

Let us consider these functions in more detail. It is easily to show that both functions $\mathcal{W}_{+}(x)$ and $\tilde{\mathcal{W}}_{+}(x)$ are negative at the infinity and positive at the minus infinity. Explicit calculations show that it provides the same behaviour of the superpotentials $\mathcal{W}(x)$, $\mathcal{W}_{1}(x)$ and $\mathcal{W}_{2}(x)$ which can be obtained by substitution functions $\mathcal{W}_{+}(x)$ and $\tilde{\mathcal{W}}_{+}(x)$ into relations (24). We shall omit explicit expressions for the superpotentials $\mathcal{W}_{1}(x)$ and $\mathcal{W}_{2}(x)$ noting only that both of them are singular while the superpotential

$$
\mathcal{W}(x)=\frac{U^{\prime}(x)\left(\mathcal{R}_{0}(x)-1\right)}{2(2 \epsilon+U(x))}+\frac{8 \epsilon \epsilon_{1}-U^{\prime \prime}(x)+2 U(x)\left(4\left(\epsilon_{1}-\epsilon\right)-3 U(x)\right)}{2 U^{\prime}(x) \mathcal{R}_{0}(x)}
$$

has no singularities if only the initial potential $V_{-}(x)$ is non-singular. Using superpotential $\mathcal{W}(x)$ we can construct in a standard way the pair of the Hamiltonians $\mathcal{H}_{-}$and $\mathcal{H}_{+}$. Their properties will be very similar to that of the Hamiltonians $H_{-}$and $H_{+}$with the only difference that now Hamiltonian $\mathcal{H}_{+}$will have zero-energy ground state with corresponding eigenfunction

$$
\varphi_{0}^{+}(x)=C \exp \left(\int \mathcal{W}(x) d x\right)
$$

All the higher eigenvalues of Hamiltonians $\mathcal{H}_{+}$and $\mathcal{H}_{-}$will coincide and corresponding 
eigenfunctions will be connected as follows

$$
\begin{aligned}
\varphi_{n+1}^{+}(x) & =\frac{1}{\sqrt{2 \mathcal{E}_{n}^{-}}}\left(\frac{d}{d x}+\mathcal{W}(x)\right) \varphi_{n}^{-}(x), \\
\varphi_{n}^{-}(x) & =\frac{1}{\sqrt{2 \mathcal{E}_{n+1}^{+}}}\left(-\frac{d}{d x}+\mathcal{W}(x)\right) \varphi_{n+1}^{+}(x) .
\end{aligned}
$$

Let us consider few the simplest explicit examples. In the case of a harmonic oscillator for which all the superpotentials and distances between the energy levels read

$$
W_{n}(x)=\epsilon x, \quad \epsilon_{n}=\epsilon
$$

we get corresponding function

$$
U(x)=4 \epsilon^{2} x^{2}
$$

and we obtain

$$
\mathcal{R}_{0}(x)=R(x)=2 \epsilon x^{2} .
$$

Choosing now $\mathcal{R}(x)=-\mathcal{R}_{0}(x)$ and using $(73)$ we get

$$
\mathcal{W}(x)=-\epsilon x \frac{5+2 \epsilon x^{2}}{1+2 \epsilon x^{2}}
$$

leading to the following SUSY partner potentials

$$
\begin{aligned}
& \mathcal{V}_{-}(x)=\frac{\epsilon^{2} x^{2}}{2}+\frac{5 \epsilon}{2} \\
& \mathcal{V}_{+}(x)=\frac{\epsilon^{2} x^{2}}{2}+\frac{4 \epsilon}{1+2 \epsilon x^{2}}-\frac{8 \epsilon}{\left(1+2 \epsilon x^{2}\right)^{2}}+\frac{3 \epsilon}{2}
\end{aligned}
$$

Thus we have obtained the exactly solvable potential (81) which is a SUSY partner to the harmonic oscillator potential (80). This potential (81) after substituting $\epsilon=b^{2} / 2$ coincides with potential (63) obtained in the section 3. Let us remind that the potential $\mathcal{V}_{+}(x)$ has zero-energy level with corresponding wave function (74). Therefore we can now treat the potentials $\mathcal{V}_{-}(x)$ and $\mathcal{V}_{+}(x)$ as $V_{+}(x)$ and $V_{-}(x)$ respectively, that is $V_{+}(x)=\mathcal{V}_{-}(x)$ and $V_{-}(x)=\mathcal{V}_{+}(x)$. The superpotential corresponding to the pair $V_{-}(x)$ and $V_{+}(x)$ is $W(x)=-\mathcal{W}(x)$. Because the upper SUSY partner $V_{+}(x)$ is a harmonic oscillator we can 
easily build up all the hierarchy of superpotentials satisfying equations (21):

$$
W_{0}(x)=\epsilon x \frac{5+2 \epsilon x^{2}}{1+2 \epsilon x^{2}}, \quad W_{n}(x)=\epsilon x, \quad n=1,2, \ldots
$$

The corresponding distances between the energy levels read

$$
\epsilon_{0}=3 \epsilon, \quad \epsilon_{n}=\epsilon, \quad n=1,2, \ldots
$$

The obtained potential (81) can be used for constructing another exactly solvable potential. Starting from the first three superpotentials (82) we obtain in the same way as before the next pair of potentials

$$
\begin{aligned}
& \mathcal{V}_{-}(x)=\frac{\epsilon^{2} x^{2}}{2}+\frac{9 \epsilon}{2} \\
& \mathcal{V}_{+}(x)=\frac{\epsilon^{2} x^{2}}{2}+\frac{8 \epsilon\left(2 \epsilon x^{2}-3\right)}{3+12 \epsilon x^{2}+4 \epsilon^{2} x^{4}}+\frac{384 \epsilon^{2} x^{2}}{\left(3+12 \epsilon x^{2}+4 \epsilon^{2} x^{4}\right)^{2}}+\frac{7 \epsilon}{2}
\end{aligned}
$$

Repeating this procedure many times we obtain the following pairs of SUSY partner potentials

$$
\begin{aligned}
V_{+}(n, x)=\mathcal{V}_{-}(n, x) & =\frac{\epsilon^{2} x^{2}}{2}+\left(2 n-\frac{1}{2}\right) \epsilon \\
V_{-}(n, x)=\mathcal{V}_{+}(n, x) & =\frac{\epsilon^{2} x^{2}}{2}+8 \epsilon n(2 n-1) \frac{H_{2 n-2}(i \sqrt{\epsilon} x)}{H_{2 n}(i \sqrt{\epsilon} x)} \\
& -16 \epsilon n^{2}\left(\frac{H_{2 n-1}(i \sqrt{\epsilon} x)}{H_{2 n}(i \sqrt{\epsilon} x)}\right)^{2}+\frac{(4 n-1) \epsilon}{2}
\end{aligned}
$$

where $H_{n}(x)$ - Hermite polynomial. Note that the case $n=1$ in the potential (87) corresponds to (81) and the case $n=2$ corresponds to (85). The potentials $V_{-}(n, x)$ (87) are just the special cases of the SUSY partner potential of a harmonic oscillator obtained by Sukumar [21] and they were previously obtained by Bagrov and Samsonov [22, 23] via the Darboux method and latter by Junker and Roy [33] within the SUSY approach.

The application of the same procedure for the Morse and Rosen-Morse potentials as well as for radial harmonic oscillator and hydrogen atom provides chains of exactly solvable potentials. All these potentials are just special cases of the potentials obtained in [33] by Junker and Roy. Nevertheless they seem to be interesting because all of them are expressed in terms of the elementary functions only. For example starting from the Morse potential for which corresponding superpotentials and distances between the energy levels read

$$
W_{n}(x)=\epsilon+1 / 2-n-e^{-x}, \quad \epsilon_{n}=\epsilon-n,
$$


we construct the function

$$
U(x)=4\left(\epsilon-e^{-x}\right)\left(\epsilon-1-e^{-x}\right) .
$$

An explicit calculations show us that we should take function $\mathcal{R}_{0}(x)$ in the form

$$
\mathcal{R}_{0}(x)=\frac{4 e^{-2 x}+6(1-2 \epsilon) e^{-x}+3(1+4 \epsilon(\epsilon-1))-2 \epsilon\left(1-3 \epsilon+2 \epsilon^{2}\right) e^{x}}{2 e^{-x}+(1-2 \epsilon)}
$$

to reproduce Morse potential. Function $\mathcal{R}_{0}(x)$ does not coincide with $R(x)$ in this case, the former is negative within the interval limited by zeros of the expression (90). Following the same procedure as in the case of harmonic oscillator we obtain such a sequence of potentials

$$
\begin{aligned}
V_{-}(0, x) & =\frac{(1+2 \epsilon)^{2}}{8}+\frac{e^{-2 x}-2(\epsilon+1) e^{-x}}{2} \\
V_{-}(1, x) & =\frac{(1+2 \epsilon)^{2}}{8}+\frac{e^{-2 x}-2(\epsilon-1) e^{-x}}{2} \\
& +\frac{2\left(\epsilon(2 \epsilon-1) e^{x}-2\right)}{\epsilon\left(2-2(2 \epsilon-1) e^{x}+\epsilon(2 \epsilon-1) e^{2 x}\right)}-\frac{8\left((2 \epsilon-1) e^{x}-1\right)}{\epsilon\left(2-2(2 \epsilon-1) e^{x}+\epsilon(2 \epsilon-1) e^{2 x}\right)^{2}} \\
V_{-}(2, x) & =\frac{(1+2 \epsilon)^{2}}{8}+\frac{e^{-2 x}-2(\epsilon-3) e^{-x}}{2} \\
& +\frac{(2 \epsilon-3)\left(8 e^{x}-(\epsilon-1)\left(48 e^{2 x}-(2 \epsilon-1)\left(36 e^{3 x}-16 \epsilon e^{4 x}\right)\right)\right)}{4-(2 \epsilon-3)\left(8 e^{x}-(\epsilon-1)\left(12 e^{2 x}-(2 \epsilon-1)\left(4 e^{3 x}-\epsilon e^{4 x}\right)\right)\right)} \\
& +\frac{\left((2 \epsilon-3)\left(8 e^{x}-(\epsilon-1)\left(24 e^{2 x}-(2 \epsilon-1)\left(12 e^{3 x}-\epsilon 4 e^{4 x}\right)\right)\right)\right)^{2}}{\left(4-(2 \epsilon-3)\left(8 e^{x}-(\epsilon-1)\left(12 e^{2 x}-(2 \epsilon-1)\left(4 e^{3 x}-\epsilon e^{4 x}\right)\right)\right)\right)^{2}} .
\end{aligned}
$$

Corresponding SUSY partners are following Morse potentials

$$
V_{+}(n, x)=\frac{(1+2 \epsilon)^{2}}{8}+\frac{e^{-2 x}-2(\epsilon-2 n) e^{-x}}{2}
$$

We can proceed with this procedure as long as necessary.

The most interesting fact is that at each step of the suggested procedure for the all mentioned above potentials

$$
\mathcal{H}_{-}=H_{+}^{(2)}+\epsilon+\epsilon_{1} .
$$

One can easily check that due to the connection (11) and (20)

$$
E_{n}^{(2)+}=E_{n+1}^{(2)-}=E_{n+1}^{(1)+}-\epsilon_{1}=E_{n+2}^{+}-\epsilon_{1}-\epsilon=E_{n+3}^{-}-\epsilon_{1}-\epsilon .
$$

and therefore the energy levels of the Hamiltonian $\mathcal{H}_{-}$coincide with that of the Hamilto- 
nian $H_{-}$saving the three lowest levels of the latter which are not present in the spectrum of the former

$$
\mathcal{E}_{n}^{-}=E_{n+3}^{-}, \quad n=0,1, \ldots
$$

It gives us immediately the energy spectrum of the new Hamiltonian $\mathcal{H}_{+}$

$$
\mathcal{E}_{0}^{-}=0, \quad \mathcal{E}_{n}^{-}=E_{n+2}^{-}, \quad n=1,2, \ldots
$$

Besides the connection (95) allows us to obtain easily all the eigenfunctions of the excited states for the new exactly solvable potential $\mathcal{H}_{+}$. Using relation (75) we obtain

$$
\varphi_{n}^{+}(x)=C_{n}^{+}\left(\frac{d}{d x}+\mathcal{W}(x)\right) \psi_{n-1}^{(2)+}(x), \quad n=1,2, \ldots
$$

Thus for all mentioned exactly solvable potentials we can construct a sequence of Hamiltonians $H_{n}=-\frac{1}{2} \frac{d^{2}}{d x^{2}}+V(n, x)$ with the same energy levels as $H_{0}$ except the $2 n$ lowest excited states of the latter. All of them possess zero-energy ground state.

\section{Conclusions}

We have obtained a general solution of the set of equations (21) for $N=2$ given by the expressions (31) and (24) $(n=0,1)$. Thus we can write down explicit expressions for superpotentials $W_{0}(x), W_{1}(x)$ and $W_{2}(x)$ and afterwards for potential $V_{-}^{(0)}(x)=$ $\left(W_{0}^{2}(x)-W_{0}^{\prime}(x)\right) / 2$ being just a general expression for a QES potential with three explicitly known eigenstates. General expressions for the corresponding eigenfunctions are also presented (9), (35). The QES potential is expressed in terms of the distances $\epsilon, \epsilon_{1}$ between neighbouring energy levels and an arbitrary function $U(x)$. To ensure nonsingularity of the potential we need to put a number of limitations on the function $U(x)$. Using this expression we have obtained some new QES potentials. In special cases our potentials reproduce those studied earlier.

There obviously arises a question if the suggested scheme could be generalized to construct QES potentials with the number of explicitly known eigenstates large than three. In such a case we have the set of equations (25) consisting of $N-2$ equations. Let us remind that $N$ is number of excited states of QES potential which we would like to construct. In order to reduce this set of equations we can proceed with the scheme described in section 3 (eqs. (21)-(31)). The solution of each equation of the set (25) can be written down in the form (31), where $W_{+}(x)$ is replaced by $W_{+}^{(n)}(x), \tilde{W}_{+}(x)$ is replaced by $W_{+}^{(n+1)}(x)$ and $U_{n}(x)=W_{+}^{(n)}(x) W_{+}^{(n+1)}(x)$ is instead of $U(x)$. Two neighbouring equations

of the set (25) yield us two different expressions for the same function $W_{+}^{(n)}$ in terms of the functions $U_{n}(x)$ and $U_{n+1}(x)$ correspondingly. Thus we obtain following set of equations for functions $U_{n}(x)$

$$
\frac{U_{n}^{\prime}(x)\left(1+\mathcal{R}_{n}(x)\right)}{2\left(U_{n}(x)+2 \epsilon_{n}\right)}=\frac{2 U_{n+1}(x)\left(U_{n+1}(x)+2 \epsilon_{n+1}\right)}{U_{n+1}^{\prime}(x)\left(1+\mathcal{R}_{n+1}(x)\right)}, \quad n=0, \ldots, N-3,
$$


where

$$
\mathcal{R}_{n}(x)= \pm \sqrt{1+4 \frac{U_{n}(x)\left(U_{n}(x)+2 \epsilon_{n}\right)\left(U_{n}(x)-2 \epsilon_{n+1}\right)}{U_{n}^{\prime}(x)^{2}}} .
$$

Number of equations in this set is one less than number of equation in the set (25) for functions $W_{+}^{(n)}(x)$ and correspondingly it is two less than the number of the initial equations (21) for surperpotentials. In the case $N=3$ we have just one equation which we need to solve to construct QES potentials with four known eigenstates. Thus one can see that the obtaining of general expression for QES potentials with more than three explicitly known eigenstates is essentially more complicated.

Another point is that the suggested scheme allows one to construct in a simple way the sequences of SUSY partner potentials of exactly solvable ones. At each step we obtain a new exactly solvable potential with identical spectrum, apart from missing two the lowest excited states. Note that this approach in contrast to method used in [20 23, 32, 33] does not require knowledge of general solution of corresponding Schrödinger equation for initial potential. It would be interesting to apply the same approach to the known QES potentials with explicitly known $N$ eigenstates to construct new QES potentials with picked out two the lowest excited states. But the latter case is more complicated than the case of shape-invariant potentials and it will be the subject of a separate paper.

\section{References}

[1] V. Singh, S. N. Biswas, K. Dutta, Phys. Rev. D 18 (1978) 1901.

[2] G. P. Flessas, Phys. Lett. A 72 (1979) 289.

[3] M. Razavy, Am. J. Phys. 48 (1980) 285; Phys. Lett A 82 (1981) 7.

[4] A. Khare, Phys. Lett. A 83 (1981) 237.

[5] O. B. Zaslavskii, V. V. Ul'yanov, V. M. Tsukernik, Fiz. Nizk. Temp. 9 (1983) 511.

[6] O. B. Zaslavsky, V. V. Ul'yanov, Zh. Eksp. Teor. Fiz. 87 (1984) 1724.

[7] A. V. Turbiner, A. G. Ushveridze, Phys. Lett. A 126 (1987) 181.

[8] A. V. Turbiner, Commun. Math. Phys. 118 (1988) 467.

[9] M. A. Shifman, Int. Jour. Mod. Phys. A 4 (1989) 2897.

[10] D. P. Jatkar, C. Nagaraja Kumar, A. Khare, Phys. Lett. A 142 (1989) 200.

[11] P. Roy, Y. P. Varshni, Mod. Phys. Lett. A 6 (1991) 1257.

[12] A. Gangopadhyaya, A. Khare, U. P. Sukhatme, Phys. Lett. A 208 (1995) 261.

[13] A. G. Ushveridze, Quasi-exactly solvable models in quantum mechanics, (Institute of Physics Publishing, Bristol 1994).

[14] B. Bagchi, C. Quesne, Phys. Lett. A 230 (1997) 1.

[15] F. Finkel, A. González-López, M. A. Rodríguez, J. Phys. A 30 (1997) 6879 
[16] V. V. Ulyanov, O. B. Zaslavskii, J. V. Vasilevskaya, Fiz. Nizk. Temp. 23 (1997) 110.

[17] Yu. V. Vasilevskaya, V. V. Ulyanov, Ukr. Fiz. Zh. 43 (1998) 363.

[18] A. Khare, B. P. Mandal, Phys. Lett. A 239 (1998) 197.

[19] C. M. Bender, S. Boettcher, J. Phys. A 31 (1998) L273.

[20] C. V. Sukumar, J. Phys. A 18 (1985), L57.

[21] C. V. Sukumar, J. Phys. A 18 (1985), 2917.

[22] V. G. Bagrov, B. F. Samsonov, Teor. Mat. Fiz. 104 (1995), 356.

[23] V. G. Bagrov, B. F. Samsonov, J. Phys. A 29 (1996), 1011.

[24] D. J. Fernández C., M. L. Glasser, L. M. Nieto, Phys. Lett. A 240 (1998) 15.

[25] D. J. Fernández C., V. Hussin, B. Mielnik, Phys. Lett. A 244 (1998) 309.

[26] A. Lahiri, P. K. Roy and B. Bagchi, Int. J. Mod. Phys. A 5 (1990) 1383.

[27] F. Cooper, A. Khare, U. Sukhatme, Phys. Rep. 251 (1995) 267.

[28] V. M. Tkachuk, Phys. Lett. A 245 (1998) 177 [preprint quant-ph/9801021 (1998)].

[29] L. E. Gendenshteyn, Pisma Zh. Eksp. Teor.Fiz. 38 (1983) 299.

[30] A. de Souza Dutra, Phys. Rev. A 47 (1993) R2435.

[31] J. Beckers, N. Debergh, A. G. Nikitin, Mod. Phys. Lett. A 8 (1993) 435.

[32] P. Roy, G. Junker, Phys. Lett. A 232 (1997) 155.

[33] G. Junker, P. Roy, preprint quant-ph/9803024 (1998). 